\title{
Reversible photocontrol of DNA coacervation
}

Suzanne Lafon, ${ }^{[a],[b]}$ Nicolas Martin[a],

[a] Univ. Bordeaux, CNRS, Centre de Recherche Paul Pascal, UMR5031, 33600 Pessac, France

[b] Ecole Normale Supérieure d'Ulm, 75005 Paris, France

E-mail: nicolas.martin@crpp.cnrs.fr

"This is the accepted version of the following article: S. Lafon, N. Martin, Methods Enzymol., 2021, 646, 329-351, which has been published in final form at https://doi.org/10.1016/bs.mie.2020.06.013."

(c) 2021. This manuscript version is made available under the CC-BY-NC-ND 4.0 license http://creativecommons.org/licenses/by-nc-nd/4.0/

\begin{abstract}
Coacervate micro-droplets produced by liquid-liquid phase separation are increasingly used to emulate the dynamical organization of membraneless organelles found in living cells. Designing synthetic coacervates able to be formed and disassembled with improved spatiotemporal control is still challenging. In this chapter, we describe the design of photoswitchable coacervate droplets produced by phase separation of short double stranded DNA in the presence of an azobenzene cations. The droplets can be reversibly dissolved with light, which provides a new approach for the spatiotemporal regulation of coacervation. Significantly, the dynamics of light-actuated droplet formation and dissolution correlates with the capture and release of guest solutes. The reported system can find applications for the dynamic photocontrol of biomolecule compartmentalization, paving the way to the light-activated regulation of signalling pathways in artificial membraneless organelles.
\end{abstract}

Keywords: photoswitchable coacervates - liquid-liquid phase separation - azobenzene - DNA • artificial membraneless organelles $\cdot$ dynamic protocells

\section{Introduction}

Membraneless organelles (MLOs) formed by liquid-liquid phase separation of proteins and RNA play a key role in the organization of intracellular contents and reactions (Hyman, Weber, \& Jülicher, 2014; Brangwynne, Tompa, \& Pappu, 2015). These biomolecular condensates include nucleoli that synthesize ribosome subunits, centrosomes that nucleate microtubules, and stress granules that segregate RNA under stress conditions. Crucial to the functions of MLOs is their highly dynamic nature that allows them to transiently compartmentalize biomolecules in cells (Wheeler, \& Hyman, 2018). In particular, the formation and dissolution, localization, size and number of MLOs rely on intracellular concentration profiles of phase separating species, which are dynamically regulated by biochemical reactions (e.g. DNA translation and transcription reactions, and posttranslational modifications) (Nott et al., 2015; Shin, \& Brangwynne, 2017; Rai, Chen, Selbach, \& Pelkmans, 2018; Berry, Brangwynne, \& Haataja, 2018; Hondele et al., 2019).

Recent efforts to design synthetic coacervate droplets able to recapitulate key features of membraneless organelles in vitro have been made. Coacervates micro-droplets are typically produced by associative liquid-liquid phase separation between oppositely charged polyions or surfactants. These liquid-like droplets provide simple models to rationalize the underlying physicochemical rules of biological phase separation (Perry, 2019), reproduce fundamental functions of biomolecular condensates (Martin, Li, \& Mann, 2016; Nakashima, Vibhute, \& Spruijt, 2019), and demonstrate primitive forms of compartmentalization ("protocells") (Crowe, \& Keating, 2018; Martin, 2019a). Dynamic aspects are also increasingly considered when designing synthetic coacervates as models of membraneless organelles and protocells (Martin, 2019a). Owing to the weak molecular interactions involved, coacervate droplets dynamically respond to environmental cues; for 
instance, they are readily formed and dissolved by changes in temperature or $\mathrm{pH}$ (Douliez et al., 2017; Martin et al., 2018; Love et al., 2020). Biochemical reactions have also recently been exploited to control the formation and dissolution of coacervate droplets, including phosphorylation/dephosphorylation reactions (Aumiller Jr, \& Keating, 2016; Nakashima, Baaji, \& Spruijt, 2018) and polynucleotide synthesis (Deshpande et al., 2019). Improved spatiotemporal control over the formation and dissolution of synthetic coacervates would be highly beneficial to emulate the dynamic regulation of biomolecular condensates: new tools for the fast and local actuation of liquid-liquid phase separation need thus to be developed.

Within this context, optogenetic approaches have recently emerged to activate the formation of membraneless organelles with light in cellulo (Shin et al., 2017). Yet, experimental models of synthetic coacervates capable of being dynamically controlled with light in vitro are required. Light is indeed a bio-orthogonal tool that offers great spatiotemporal capabilities and spectral selectivity. Light is also one of the primary energy sources on Earth, making it an interesting tool to control membrane-free compartmentalization in the context of origin-of-life studies. Overall, the design of photoswicthable coacervates would open new perspectives for the spatiotemporal regulation of biomolecule compartmentalization.

\section{Photoswitchable coacervation: the azoTAB/dsDNA system}

We recently reported an approach for the reversible photocontrol of DNA coacervation in vitro using molecular azobenzene photoswitches (Martin et al. 2019b). In this system, liquid-liquid phase separation of short double stranded DNA ( $d s D N A$ ) is induced by an azobenzene-based cation, azobenzene trimethylammonium bromide (azoTAB). The overall photoswitchable process is summarized in Figure $1 \mathrm{~A}$.

Azobenzene moieties are well known to undergo trans-cis photoisomerisation under light irradiation. The resulting changes in geometry and dipolar moment at the molecular level have been exploited to control the structure and function of biomolecules. For instance, azoTAB cations have been used to control the compaction of single dsDNA chains with light, as first reported by Lee et coll. (Le Ny, \& Lee, 2006), followed by studies from Baigl et coll. (Sollogoub et al., 2008; Diguet, Mani, Geoffroy, Sollogoub, \& Baigl, 2010), who further demonstrated light-mediated regulation of cell-free gene expression via the reversible photocontrol of dsDNA condensation (Estévez-Torres et al., 2009). In these studies, long dsDNA chains were used (e.g. bacteriophage DNA and plasmids, $>1000$ base pairs) at relatively low concentrations (typically $\mu \mathrm{M}$ nucleobase concentrations). Observations pointed to a contribution of both electrostatic and hydrophobic forces in the dsDNA compaction process.

In comparison, photoswitchable coacervates are produced with relatively short $d s \mathrm{DNA}$ chains (<200 base pairs) and at higher polynucleotide concentration ( $\sim \mathrm{mM}$ nucleobase concentration) (Martin et al. 2019b). Our observation that coacervation between dsDNA and trans-azoTAB occurs close to equimolar charge ratio indicates that electrostatic interactions play an important role in the phase separation process. Additional forces involved in the process include hydrophobic interactions between azobenzene moieties (e.g. $\pi-\pi$ stacking) and partial intercalation of the planar trans-azoTAB between $d s D N A$ base pairs (Martin et al. 2019b). In comparison, cis-azoTAB has a lower affinity for $d s D N A$ because it is less hydrophobic and can no longer develop $\pi-\pi$ stacking due to its bent geometry: its unbinding from $d s D N A$ eventually leads to the dissolution of coacervate microdroplets. Overall, UV light converts the coacervate-active trans-azoTAB into the coacervatedormant cis isomer, resulting in the dissolution of coacervate droplets. Reversibly, cis-trans isomerisation under blue light favours the re-assembly of coacervate micro-droplets (Figure $1 \mathrm{~A})$. 
We have further demonstrated that the dynamics of droplet dissolution and reformation correlates with the release and capture of guest solutes: solutes sequestered within droplets are released under UV light due to coacervate disassembly, and are recaptured in the droplets that re-form under blue light. Such a photoswitchable release and capture of solutes can find applications to dynamically control the compartmentalization of biomolecules, opening new perspectives for the regulation of artificial membraneless organelle systems.

This chapter describes the methods for the formulation and reversible photocontrol of azoTAB/dsDNA coacervate micro-droplets, including the protocols for the synthesis of the cationic azoTAB photoswitch.

A

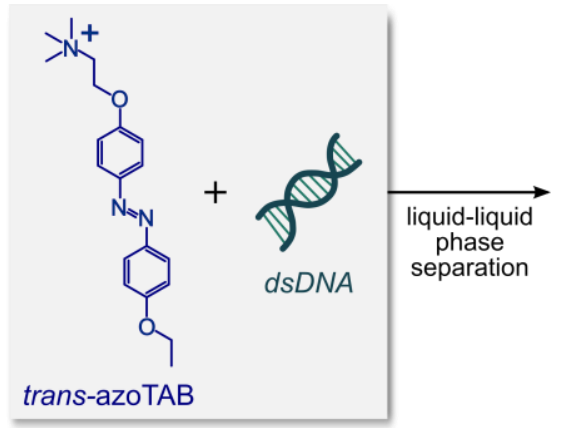

B

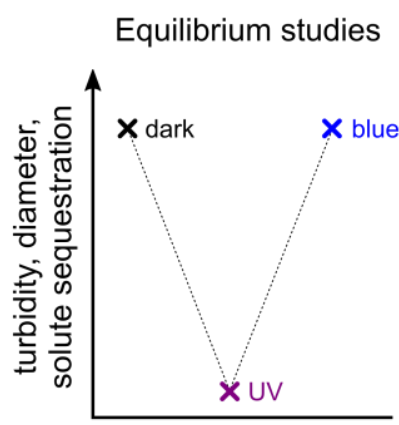

Global irradiation

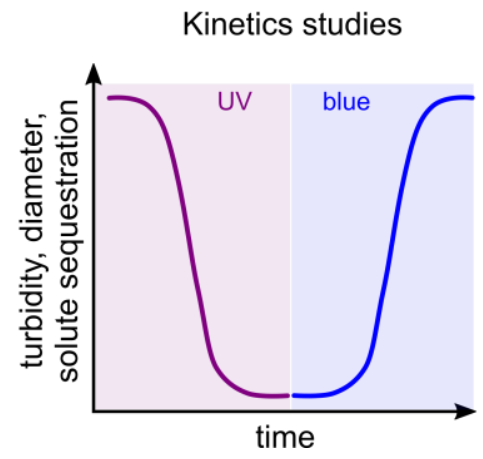

C

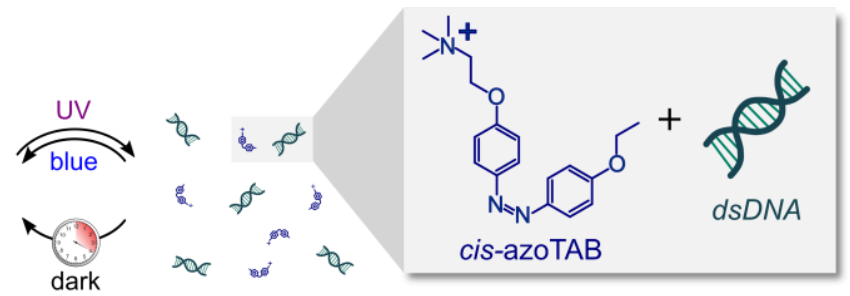

coacervate micro-droplet

Local irradiation
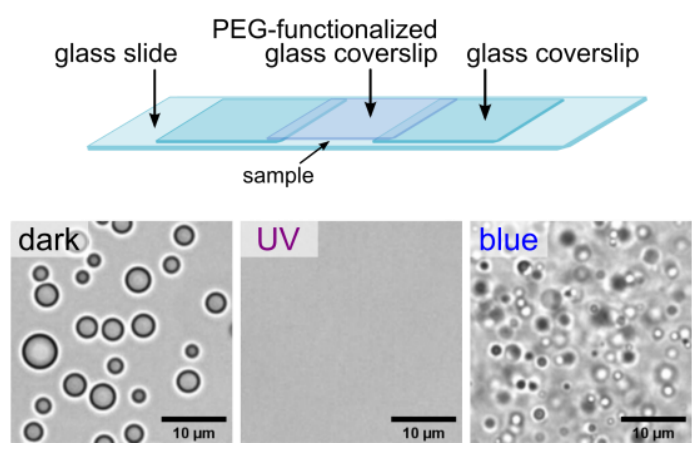

Figure 1. A, Scheme of reversible photocontrol of $d s D N A$ coacervation with azoTAB. ON/OFF phase separation can be triggered in response to UV and blue light. Temporal programming of coacervate droplets formation is achieved in the dark. B, Photoswitchable phase separation and the associated sequestration/release of solutes can be characterized under global irradiation of a droplets suspension at equilibrium (photostationary state) or via kinetics studies. C, These processes can also be monitored under local irradiation by optical microscopy. Droplets are here imaged with an oil immersion objective on an inverted microscope using a PEG-functionalized capillary chamber.

\section{Synthesis and photophysical characterization of azoTAB}

AzoTAB is produced by a three-step synthesis derived from the method originally described by Hayashita et al. (Hayashita, Kurosawa, Miyata, Tanaka, \& Igawa, 1994), and further adapted by Lee et coll. (Le Ny, \& Lee, 2006) and Baigl et coll. (Sollogoub et al., 2008; Diguet, Mani, Geoffroy, Sollogoub, \& Baigl, 2010; Estévez-Torres et al., 2009). We detail below the synthesis procedure (summarized in Figure $2 \mathrm{~A}$ ).

\subsection{Protocol for the synthesis of azoTAB}

\subsubsection{Equipment}

1. Round-bottomed flasks (two-necked, $250 \mathrm{~mL}$; single-necked, $150 \mathrm{~mL}$ ) equipped with septa

2. Magnetic stirring bar 


\section{Thermometer}

4. Stirring hotplate

5. Aluminium foil

6. $\mathrm{pH}$ test strips

7. Analytical balance

8. Ice bath; oil bath

9. Vacuum filtration set-up: filter, Büchner funnel, conic seal, Büchner flask, vacuum pump

10. Separation funnel $(50 \mathrm{~mL})$

11. Rotary evaporator

12. NMR spectrometer

A
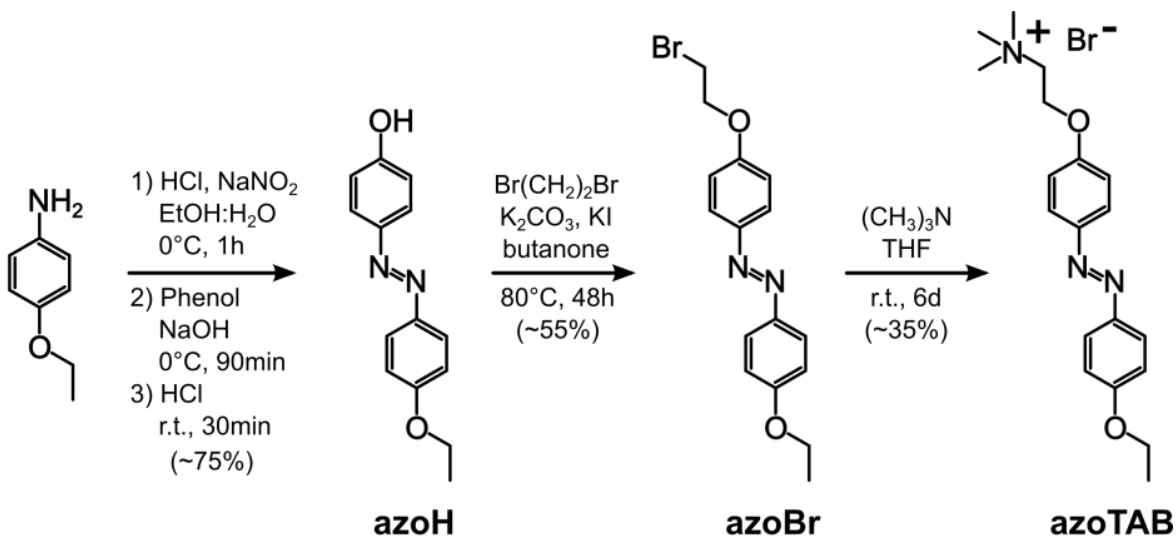

B
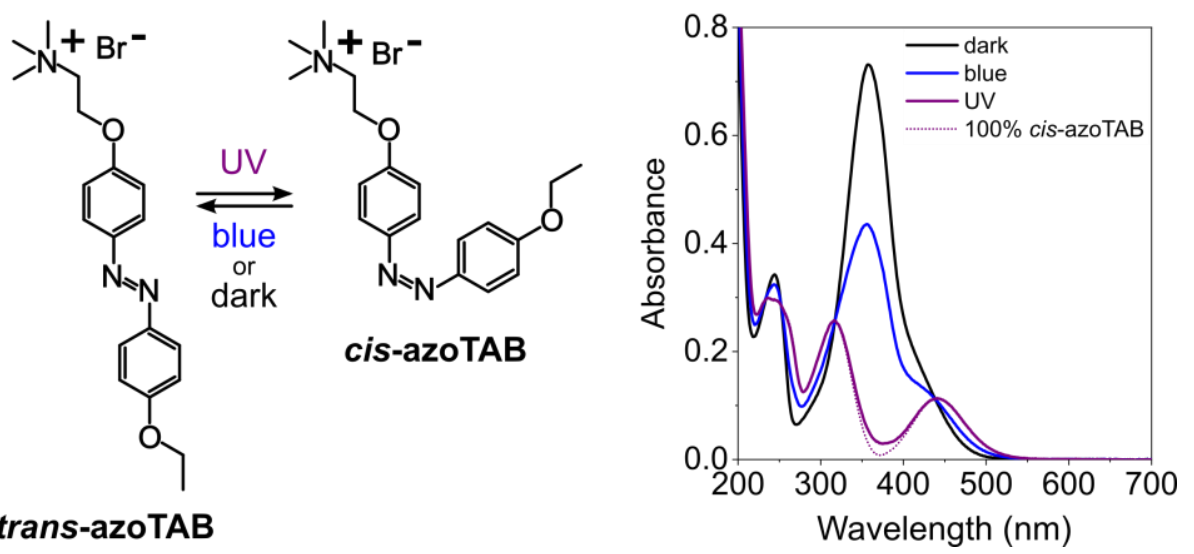

Figure 2. A, General overview of the synthesis procedure of azoTAB. B, Photophysical characterization of azoTAB isomerisation. UV/vis spectra of a $30 \mu \mathrm{M}$ azoTAB solution equilibrated in the dark (black line, corresponding to $100 \%$ trans-azoTAB), and exposed to UV (purple line) or blue (blue line) light are reported, together with the calculated spectrum for $100 \%$ cis-azoTAB.

\subsubsection{Chemicals}

Commercially available reagents were used as received. The ${ }^{1} \mathrm{H}$ and ${ }^{13} \mathrm{C}$ NMR spectra were recorded at room temperature on a $400 \mathrm{MHz}$ ECZ400 spectrometer (Jeol); chemical shifts are reported in ppm with protonated solvent as internal reference $\left({ }^{1} \mathrm{H}, \mathrm{CHCl}_{3}\right.$ in $\mathrm{CDCl}_{3} 7.26$ ppm, $\mathrm{CHD}_{2} \mathrm{SOCD}_{3}$ in $\mathrm{CD}_{3} \mathrm{SOCD}_{3} 2.49 \mathrm{ppm} ;{ }^{13} \mathrm{C},{ }^{13} \mathrm{CDCl}_{3}$ in $\mathrm{CDCl}_{3} 77.0,{ }^{13} \mathrm{CD}_{3} \mathrm{SOCD}_{3}$ in $\left.\mathrm{CD}_{3} \mathrm{SOCD}_{3} 39.7 \mathrm{ppm}\right)$; coupling constants $(\mathcal{J}$ ) are given in $\mathrm{Hz}$.

1. Para-ethoxyaniline (Sigma-Aldrich)

2. Hydrochloric acid 37\% (Sigma-Aldrich)

3. Sodium hydroxide (Sigma-Aldrich)

4. Sodium nitrite (Sigma-Aldrich)

5. Phenol (Sigma-Aldrich) 
6. 1,2-Dibromoethane (Sigma-Aldrich)

7. Potassium carbonate (Sigma-Aldrich)

8. Potassium iodide (Sigma-Aldrich)

9. 33\% Trimethylamine solution in ethanol (Sigma-Aldrich)

10. Magnesium sulfate $\left(\mathrm{MgSO}_{4}\right)$ (Sigma-Aldrich)

11. Ethanol (Sigma-Aldrich)

12. Methyl ethyl ketone (Sigma-Aldrich)

13. Dichloromethane (Sigma-Aldrich)

14. Anhydrous tetrahydrofuran (Sigma-Aldrich)

15. DMSO-d 6 and $\mathrm{CDCl}_{3}$ for NMR experiments (Sigma-Aldrich)

\subsubsection{Protocol}

All synthetic steps are performed in a fume hood.

\section{Synthesis of 4-ethoxy-4'-hydroxy-azobenzene (azoH)}

1.1. Working at $0^{\circ} \mathrm{C}$ in an ice bath, add concentrated $\mathrm{HCl}(17 \mathrm{~mL}, 37 \%$, dropwise addition) and ice $(80 \mathrm{~g})$ to a $1: 1 \mathrm{v} / \mathrm{v}$ ethanol:water solution $(160 \mathrm{~mL})$ containing para-ethoxyaniline (10.3 mL, $80 \mathrm{mmol}, 1$ equiv.) and sodium nitrite $(5.5 \mathrm{~g}, 80 \mathrm{mmol}, 1$ equiv.) in a two-necked round-bottomed flask $(250 \mathrm{~mL})$ under magnetic stirring. The temperature of the mixture can be checked with a thermometer.

1.2. Stir the mixture for $1 \mathrm{~h}$ while maintaining the temperature below $5^{\circ} \mathrm{C}$ with an ice bath.

1.3. Carefully add a solution of phenol $(7.5 \mathrm{~g}, 80 \mathrm{mmol}, 1$ equiv.) and $\mathrm{NaOH}(6.4 \mathrm{~g}, 160$ $\mathrm{mmol}, 2$ equiv.) dissolved in cold water $\left(42 \mathrm{~mL}, \sim 5^{\circ} \mathrm{C}\right)$ to the mixture and stir for 90 min while maintaining the temperature below $5^{\circ} \mathrm{C}$ with an ice bath.

1.4. Adjust the $\mathrm{pH}$ of the solution to 1 with concentrated $\mathrm{HCl}(37 \%)$ using $\mathrm{pH}$ test strips, and leave the mixture to warm to room temperature for $30 \mathrm{~min}$. A brown precipitate should form. 1.5. Recover the precipitate with vacuum filtration, wash thoroughly with water and then dry in vacuum overnight. The typical yield of the reaction is ca. $75 \%$.

1.6. Analyze the dark brown powder obtained (azoH) by NMR to yield: ${ }^{1} \mathrm{H}$ NMR (ppm, 400 $\mathrm{MHz}, \mathrm{CDCl}_{3}$ ): $\delta=7.86$ (d, 2H; Ar-H, J=8Hz), 7.82 (d, $\left.2 \mathrm{H} ; \mathrm{Ar}-\mathrm{H}, \mathrm{J}=8 \mathrm{~Hz}\right), 6.99$ (d, 2H; Ar-H, $J=8 \mathrm{~Hz}$ ), 6.94 (d, 2H; $\mathrm{Ar}-\mathrm{H}, \mathrm{J}=8 \mathrm{~Hz}$ ), 4.12 (q, 2H; $\left.\mathrm{CH}_{2}, \mathrm{~J}=6 \mathrm{~Hz}\right), 1.46 \mathrm{ppm}$ (t, $3 \mathrm{H} ; \mathrm{CH}_{3}, \mathrm{~J}=4 \mathrm{~Hz}$ ); ${ }^{13} \mathrm{C} \mathrm{NMR}$ (ppm, $400 \mathrm{MHz}, \mathrm{CDCl}_{3}$ ): $\delta=161.0$ (Ar-C), 157.9 (Ar-C), 147.1 (Ar-C), 146.8 (ArC), 124.6 (Ar-C), 124.4 (Ar-C), 115.8 (Ar-C), 114.7 (Ar-C), $63.8\left(\mathrm{CH}_{2} \mathrm{O}\right), 14.8 \mathrm{ppm}\left(\mathrm{CH}_{3}\right)$. These data are in line with the reported values for the same compound (Diguet, Mani, Geoffroy, Sollogoub, \& Baigl, 2010).

\section{Synthesis of 4-ethoxy-(4'-(2-bromoethyloxy)phenyl)azobenzene (azoBr)}

2.1. Mix azoH (2.4 g, $10 \mathrm{mmol}, 1$ equiv.), 1,2-dibromoethane (5.6 g, 3 equiv.), potassium carbonate $(2.07 \mathrm{~g}, 1.5$ equiv.) and potassium iodide $(0.083 \mathrm{~g}, 0.05$ equiv.) in $50 \mathrm{~mL}$ of methyl ethyl ketone (butanone) in a single-necked round-bottomed flask $(150 \mathrm{~mL}$ ) equipped with a condenser, and reflux $\left(\sim 80^{\circ} \mathrm{C}\right)$ for $48 \mathrm{~h}$ in the dark (use aluminium foil around the flask) under magnetic stirring.

2.2. Filter the reaction mixture hot to remove any solid impurities and salt, and wash the residue with methyl ethyl ketone.

2.3. Collect the filtrate and remove the solvent under reduced pressure by rotary evaporation.

2.4. Dissolve the obtained solid in dichloromethane $(20 \mathrm{~mL})$, wash twice with a $\mathrm{NaOH}$ solution $(1 \mathrm{M}, 2 \times 8 \mathrm{~mL})$, then twice with pure water $(2 \times 8 \mathrm{~mL})$, dry over $\mathrm{MgSO}_{4}$ and concentrate under reduced pressure to yield an orange powder.

2.5. Analyse the orange powder obtained by ${ }^{1} \mathrm{H}$ NMR (see 2.6). The product may be pure enough to be used without further purification for the next step. Alternatively, the crude 
product can be recrystallized with hot filtration from ethanol and dried under vacuum overnight. The typical yield of the reaction is ca. $55 \%$.

2.6. Analyse the orange powder obtained (azoBr) by NMR to yield: ${ }^{1} \mathrm{H} N M R$ (ppm, $400 \mathrm{MHz}$, $\mathrm{CDCl} 3$ ): $\delta=7.92$ (q, 4H; $\mathrm{Ar}-\mathrm{H}, \mathrm{J}=8.2 \mathrm{~Hz}$ ), 7.00 (dd, 4H; $\mathrm{Ar}-\mathrm{H}, \mathrm{J}=8.6 \mathrm{~Hz}$ ), 4.37 (t, $2 \mathrm{H} ; \mathrm{CH}_{2} \mathrm{O}$, $J=8 \mathrm{~Hz}$ ), 4.12 (q, 2H; $\mathrm{CH}_{2} \mathrm{O}, \mathrm{J}=6 \mathrm{~Hz}$ ), 3.67 (t, 2H; $\mathrm{CH}_{2} \mathrm{Br}, \mathrm{J}=7 \mathrm{~Hz}$ ), 1.46 ppm (t, 3H; $\mathrm{CH}_{3}$, $J=6 \mathrm{~Hz}$ ); ${ }^{13} \mathrm{C}$ NMR (ppm, $\left.400 \mathrm{MHz}, \mathrm{CDCl}_{3}\right): \delta=161.5(\mathrm{Ar}-\mathrm{C}), 160.2(\mathrm{Ar}-\mathrm{C}), 146.8(\mathrm{Ar}-\mathrm{C})$, 146.3 (Ar-C), 124.8 (Ar-C), 124.6 (Ar-C), 114.9 (Ar-C), 114.8 (Ar-C), $68.0\left(\mathrm{CH}_{2} \mathrm{O}\right), 63.9$ $\left(\mathrm{CH}_{2} \mathrm{O}\right), 28.8\left(\mathrm{CH}_{2} \mathrm{Br}\right), 14.8 \mathrm{ppm}\left(\mathrm{CH}_{3}\right)$.

\section{Synthesis of azobenzene trimethylammonium bromide (azoTAB)}

3.1. Working at room temperature, dissolve $1 \mathrm{~g}$ of azoBr $(4.4 \mathrm{mmol}, 1$ equiv.) in $80 \mathrm{~mL}$ of dry THF in a single-necked round-bottomed flask $(150 \mathrm{~mL})$, then add a $33 \%$ solution of trimethylamine in ethanol $(4.2 \mathrm{~mL}, 11.5 \mathrm{mmol}, 4$ equiv.) under magnetic stirring.

3.2. Stir the mixture for 6 days in the dark (use aluminium foil around the flask) at room temperature. A precipitate should form over time. Since trimethylamine is highly volatile, another aliquot of the $33 \%$ solution of trimethylamine in ethanol $(4.2 \mathrm{~mL}, 11.5 \mathrm{mmol}, 4$ equiv.) should be added after 3 days.

3.3. Recover the resulting orange precipitate with vacuum filtration, wash thoroughly with THF, and dry under vacuum overnight.

3.4. Analyse the orange powder obtained by ${ }^{1} \mathrm{H}$ NMR (see 3.5). The product may be pure enough to be used without further purification. Alternatively, the crude product can be recrystallized from ethanol and dried under vacuum overnight. The typical yield of the reaction is ca. $35 \%$.

3.5. Analyse the orange powder obtained (azoTAB) by NMR to yield: ${ }^{1} \mathrm{H}$ NMR (ppm, 400 $\mathrm{MHz}, \mathrm{DMSO}): \delta=7.86(\mathrm{~d}, 2 \mathrm{H} ; \mathrm{Ar}-\mathrm{H}, \mathrm{J}=8 \mathrm{~Hz}), 7.82(\mathrm{~d}, 2 \mathrm{H} ; \mathrm{Ar}-\mathrm{H}, \mathrm{J}=8 \mathrm{~Hz}), 7.17(\mathrm{~d}, 2 \mathrm{H} ; \mathrm{Ar}-\mathrm{H}$, $J=8 \mathrm{~Hz}$ ), 7.08 (d, 2H; $\mathrm{Ar}-\mathrm{H}, \mathrm{J}=8 \mathrm{~Hz}), 4.56\left(\mathrm{~m}, 2 \mathrm{H} ; \mathrm{CH}_{2} \mathrm{O}\right), 4.11\left(\mathrm{q}, 2 \mathrm{H} ; \mathrm{CH}_{2} \mathrm{O}, \mathrm{J}=6 \mathrm{~Hz}\right), 3.82$ (m, $\left.2 \mathrm{H} ; \mathrm{CH}_{2} \mathrm{~N}\right), 3.18\left(\mathrm{~s}, 9 \mathrm{H} ; \mathrm{CH}_{3} \mathrm{~N}\right), 1.35 \mathrm{ppm}\left(\mathrm{t}, 3 \mathrm{H} ; \mathrm{CH}_{3}, \mathrm{~J}=4 \mathrm{~Hz}\right) ;{ }^{13} \mathrm{C}$ NMR (ppm, $400 \mathrm{MHz}$, DMSO): $\delta=161.3$ (Ar-C), 159.9 (Ar-C), 147.1 (Ar-C), 146.4 (Ar-C), 124.7 (Ar-C), 124.5 (ArC), $115.8(\mathrm{Ar}-\mathrm{C}), 115.4(\mathrm{Ar}-\mathrm{C}), 64.5\left(\mathrm{CH}_{2} \mathrm{O}\right), 64.1\left(\mathrm{CH}_{2} \mathrm{~N}\right), 62.5\left(\mathrm{CH}_{2} \mathrm{O}\right), 53.6\left(\mathrm{CH}_{3} \mathrm{~N}\right), 15.0$ ppm $\left(\mathrm{CH}_{3}\right)$.

\subsection{Photo-isomerisation of azoTAB: equilibrium trans:cis composition}

Trans-azoTAB is the thermodynamically most stable isomer due to its planar geometry and aromaticity, therefore a solution of azoTAB kept in the dark for several days contains $100 \%$ of trans-azoTAB. Exposure of the solution to light will result in a mixture of cis and trans isomers, with an equilibrium composition that depends on the irradiation wavelength (Hayashita, Kurosawa, Miyata, Tanaka, \& Igawa, 1994; Diguet, Mani, Geoffroy, Sollogoub, \& Baigl, 2010; Chevallier et al., 2011). Typically, trans-cis isomerisation of azoTAB is induced by irradiation at $365 \mathrm{~nm}$ (a value close to the wavelength of the $\pi-\pi^{*}$ absorption peak), and results in almost quantitative conversion of trans-azoTAB into the cis isomer. In comparison, the reversible cis-trans isomerisation is induced by irradiation at 450 $\mathrm{nm}$ ( $\mathrm{n}-\mathrm{m}^{*}$ absorption peak), and produces a mixture of trans and cis-azoTAB at the photostationary state (with a small excess of the trans isomer) because both isomers absorb light at $450 \mathrm{~nm}$.

The composition of trans and cis isomers under UV and blue light can be easily determined by UV/vis spectrophotometry, and should be assessed before producing coacervates. These studies, detailed below, also allow to verify that the light sources subsequently used to control photoswitchable coacervates are effective to induce azoTAB photo-isomerisation.

Different light sources can be used to isomerise azoTAB, including LEDs and benchtop UV lamps for global irradiation, or light sources directly installed on a microscope for local 
irradiation. A power per unit area of a few $\mathrm{mW} . \mathrm{cm}^{-2}$ is sufficient to induce azoTAB isomerisation. The power of the identified light source can be assessed using a power-meter when available, in which case the sensor of the power-meter should be located at the position of the sample.

\subsubsection{Equipment}

1. Analytical balance

2. Ultrasound bath

3. Heating bath or Eppendorf thermomixer

4. $\mathrm{pH}$-meter

5. Micro-pipettes and pipette tips

6. Eppendorf tubes

7. $3 \mathrm{~mL}$ quartz cuvette

8. Small magnetic stirring bar

9. UV/vis spectrophotometer equipped with magnetic stirring

10. UV (365 nm) and blue (450 nm) LEDs. In the original study, LEDs from Thorlabs were used Erreur ! Signet non défini. (365 nm: reference M365D2, $1150 \mathrm{~mW} ; 450 \mathrm{~nm}$ : reference M450D3, $1850 \mathrm{~mW}$ ).

\subsubsection{Protocols and analysis}

\section{Preparation of a $100 \mathrm{mM}$ stock solution of trans-azoTAB}

1. Dissolve $40.8 \mathrm{mg}$ of synthesized azoTAB powder (MazoTAB $=408.3 \mathrm{~g} / \mathrm{mol}$ ) in $950 \mu \mathrm{L}$ of Milli-Q water in an Eppendorf tube. Sonication, vortexing and heating $\left(\sim 65^{\circ} \mathrm{C}\right)$ can be used to favour solubilisation. The resulting orange solution should be clear.

2. Check the $\mathrm{pH}$ with a calibrated $\mathrm{pH}$-meter and adjust if necessary to $\mathrm{pH} 8$ with $0.1 \mathrm{M} \mathrm{HCl}$ or $\mathrm{NaOH}$ solutions, then fill to $1 \mathrm{~mL}$ with Milli-Q water to make a $100 \mathrm{mM}$ azoTAB stock solution.

3. Store in the dark at room temperature for at least 3 days before use to ensure that all azoTAB molecules have relaxed to the trans isomer. Alternatively, the stock solution can be heated to $65^{\circ} \mathrm{C}$ for at least 1 hour to accelerate the cis-trans thermal relaxation using a heating bath or an Eppendorf thermomixer, then let to cool to room temperature in the dark. 4 Store at room temperature in the dark (e.g. by wrapping the Eppendorf tube in aluminium foil). The trans-azoTAB stock solution is stable for extended periods of time (months). The solution can be sonicated, vortexed and heated to re-dissolve any precipitate that may form over time (e.g. if the temperature in the room is too cold).

\section{$\mathrm{UV} / \mathrm{vis}$ measurements in the dark, and after UV and blue light irradiation}

1. After baseline acquisition using pure water, record the UV/vis spectrum of a $30 \mu \mathrm{M}$ transazoTAB solution prepared by dilution of the $100 \mathrm{mM}$ stock solution with Milli-Q water in a quartz cuvette (wavelength range: $200-600 \mathrm{~nm}$ ). It is important to perform this experiment in the dark (e.g. using foil, or in a dark room to prepare the solution) to make sure that all azoTAB molecules remain in their trans configuration.

2. Irradiate the cuvette with a UV light source $(365 \mathrm{~nm})$ under magnetic stirring for at least 5 minutes (the less intense the source, the longer the irradiation should be to ensure that equilibrium is reached). The irradiation can be performed from the top or the side of the cuvette using a LED (from the top, the irradiation can be performed directly in the UV/vis spectrophotometer if equipped with magnetic stirring).

3. Turn off the light source and immediately acquire the UV/vis spectrum of the UVequilibrated solution (wavelength range: $200-600 \mathrm{~nm}$ ). 
4. Repeat the same procedure with blue light irradiation: irradiate the cuvette with a blue light source $(450 \mathrm{~nm})$ under magnetic stirring for at least 5 minutes, then turn off the light source and acquire the UV/vis spectrum of the blue-adapted solution (wavelength range: 200-600 nm).

\section{Analysis: determination of the trans:cis composition at the photostationary state}

The UV/vis spectra acquired on the same azoTAB solution irradiated with UV and blue light are used to determine the fraction of trans and cis isomers at equilibrium. We assume that the dark-adapted solution contains $100 \%$ trans-azoTAB. This can be confirmed by checking that the UV/vis spectrum of the dark-adapted sample does not change over time.

Absorbances of the dark-, UV- and blue-adapted samples are considered to vary in proportion to the trans:cis composition, according to the following equations (Chevallier et al., 2011; Martin et al., 2017):

$A^{\text {dark }}(\lambda)=\varepsilon_{\text {trans }}(\lambda) . c . l=A_{\text {trans }}(\lambda)$

$A^{U V}(\lambda)=\left[\varepsilon_{\text {trans }}(\lambda) \cdot x_{\text {trans }}^{U V}+\varepsilon_{\text {cis }}(\lambda) \cdot x_{\text {cis }}^{U V}\right] . c . l=A_{\text {trans }}(\lambda) \cdot x_{\text {trans }}^{U V}+A_{\text {cis }}(\lambda) \cdot x_{\text {cis }}^{U V}$

$A^{\text {blue }}(\lambda)=\left[\varepsilon_{\text {trans }}(\lambda) \cdot x_{\text {trans }}^{\text {blue }}+\varepsilon_{\text {cis }}(\lambda) \cdot x_{\text {cis }}^{\text {blue }}\right] . c . l=A_{\text {trans }}(\lambda) \cdot x_{\text {trans }}^{\text {blue }}+A_{\text {cis }}(\lambda) \cdot x_{\text {cis }}^{\text {blue }}$

where $A^{\text {dark }}(\lambda), A^{U V}(\lambda)$ and $A^{\text {blue }}(\lambda)$ are the wavelength-dependent absorbances of the dark-, UV- and blue-adapted solutions, respectively; $l$ is the pathlength; $x_{\text {trans }}^{U V}$ and $x_{\text {cis }}^{U V}$ are the fractions of trans and cis isomers in the UV-adapted sample; $x_{\text {trans }}^{\text {blue }}$ and $x_{\text {cis }}^{\text {blue }}$ are the fractions of trans and cis isomers in the blue-adapted sample; $\varepsilon_{\text {trans }}(\lambda)$ and $A_{\text {trans }}(\lambda)$ are the wavelength-dependent extinction coefficient and absorbance of trans-azoTAB, respectively; $\varepsilon_{\text {cis }}(\lambda)$ and $A_{\text {cis }}(\lambda)$ are the wavelength-dependent extinction coefficient and absorbance of cis-azoTAB, respectively; and $c$ is the total azoTAB concentration.

The values of $\varepsilon_{\text {trans }}(\lambda)$ are obtained from the dark-adapted sample using Eq. (1). To obtain the values of $\varepsilon_{\text {cis }}(\lambda)$, and in turn the trans:cis composition under UV and blue light, an iterative process is used. Initial $x_{\text {trans }}^{U V}$ and $x_{\text {trans }}^{\text {blue }}$ values are calculated by assuming $\varepsilon_{\text {cis }}\left(\lambda_{\text {min }}^{U V}\right) \cdot x_{\text {cis }}^{i}<<\varepsilon_{\text {trans }}\left(\lambda_{\text {min }}^{U V}\right) \cdot x_{\text {trans }}^{i}$ in Eqs (2) $(i=U V)$ and (3) $(i=b l u e)$, where $\lambda_{\text {min }}^{U V}$ is the wavelength at the minimum of absorption of the UV-adapted sample in the range $300<\lambda<$ $450 \mathrm{~nm}$ (here, $\lambda_{\min }^{U V}=375 \mathrm{~nm}$ ). At $\lambda_{\min }^{U V}$, we can thus estimate $x_{\text {trans }}^{U V}$ and $x_{\text {trans }}^{\text {blue }}$ as:

$$
\begin{aligned}
& x_{\text {trans }}^{U V}=\frac{A^{U V}\left(\lambda_{\min }^{U V}\right)}{A^{\text {dark }}\left(\lambda_{\min }^{U V}\right)} \\
& x_{\text {trans }}^{\text {blue }}=\frac{A^{\text {blue }}\left(\lambda_{\min }^{U V}\right)}{A^{\text {dark }}\left(\lambda_{\min }^{U V}\right)}
\end{aligned}
$$

The absorbance spectrum of the trans isomer weighed by the calculated $x_{\text {trans }}^{U V}$ and $x_{\text {trans }}^{\text {blue }}$ values is subtracted from the UV- and blue-adapted spectra, and normalized by $x_{c i s}^{U V}$ and $x_{\text {cis }}^{\text {blue }}$, respectively, to give a putative absorbance spectrum for the cis isomer, using Eqs. (2) and (3). An iterative process is then used to minimize the difference between the spectra calculated for the cis isomer from the UV-and the blue-adapted solutions in the range 300 $\mathrm{nm}<\lambda<450 \mathrm{~nm}$. At the end of the process, the two calculated spectra for the cis isomer converge to a single one shown in Figure 2B. Typical values of $x_{\text {trans }}$ and $x_{\text {cis }}$ obtained after this process are listed below.

\begin{tabular}{ccc}
\hline & UV $(365 \mathrm{~nm})$ & Blue $(450 \mathrm{~nm})$ \\
\hline$x_{\text {trans }}$ & $4 \%$ & $58 \%$ \\
$x_{\text {cis }}$ & $96 \%$ & $42 \%$ \\
\hline
\end{tabular}

\section{Photoswitchable DNA coacervation}

\subsection{Preparation of trans-azoTAB/dsDNA coacervates}


Trans-azoTAB/dsDNA coacervate micro-droplets are prepared at equimolar charge ratio, typically at $5 \mathrm{mM}$ final concentration of trans-azoTAB and dsDNA (nucleobase concentration). Short DNA strands are used (typically <200bp) because longer strands ( 2000bp) form solid-like precipitates in the presence of trans-azoTAB.

In our previous study, we further observed that droplets formed in the absence of salt were small, with an average hydrodynamic diameter of $\sim 200 \mathrm{~nm}$ as determined by dynamic light scattering (Martin et al., 2019b). Increasing the ionic strength to $100 \mathrm{mM}$ using sodium chloride $(\mathrm{NaCl})$ favoured the formation of micrometre-sized droplets, consistent with the enhancement of coacervation reported in the literature upon addition of small amounts of salt (Perry, Li, Priftis, Leon, \& Tirrell, 2014). In comparison, coacervation was prevented at much higher ionic strength $(>500 \mathrm{mM} \mathrm{NaCl})$, as expected from screening of attractive electrostatic interactions between trans-azoTAB and dsDNA. We therefore fixed the $\mathrm{NaCl}$ concentration to $100 \mathrm{mM}$ to favour the formation of the largest coacervate droplets. We detail below the preparation of a suspension of such coacervate micro-droplets.

\subsubsection{Equipment}

1. Analytical balance

2. $\mathrm{pH}$-meter

3. Micro-pipettes and pipette tips

3. Eppendorf tubes

\subsubsection{Chemicals}

1. Deoxyribonucleic acid, low molecular weight from salmon sperm, dsDNA (Sigma-Aldrich, reference 31149)

2. Synthesized azoTAB (see 3.1.)

3. Sodium chloride $(\mathrm{NaCl})$

4. $\mathrm{NaOH}$ and $\mathrm{HCl}$ solutions $\left(0.1\right.$ and $\left.1 \mathrm{~mol}^{-\mathrm{L}^{-1}}\right)$

\subsubsection{Protocol}

\section{Preparation of a $100 \mathrm{mM}$ dsDNA stock solution}

1. Dissolve $30.4 \mathrm{mg}$ of $d s \mathrm{DNA}(0.1 \mathrm{mmol}$ nucleobases, considering an average Mnucleobase of $303.7 \mathrm{~g} \cdot \mathrm{mol}^{-1}$ ) in $700 \mu \mathrm{L}$ of milli-Q water containing $40 \mu \mathrm{L}$ of $\mathrm{NaOH} 1 \mathrm{~mol}^{-\mathrm{L}^{-1}}$ and gently mix by pipetting until the DNA is fully dissolved.

2. Check the $\mathrm{pH}$ and adjust to $\mathrm{pH} 8$ by adding small volumes of a 1 mol.L-1 $\mathrm{NaOH}$ solution, then fill to $1 \mathrm{~mL}$ with Milli-Q water to make a $100 \mathrm{mM}$ stock dsDNA solution (nucleobases concentration).

3. Split the solution into aliquots and store in the freezer at $-20^{\circ} \mathrm{C}$ until use.

\section{Preparation of trans-azoTAB/dsDNA coacervates}

1. Mix in the following order aliquots of stock solutions of trans-azoTAB (100 mM, see 3.2.2.), $\mathrm{NaCl}(2 \mathrm{M})$ and dsDNA (100 mM nucleobases) in Milli-Q water in an Eppendorf tube to reach final concentrations of $5 \mathrm{mM}$ trans-azoTAB, $5 \mathrm{mM}$ dsDNA (nucleobase concentration) and $100 \mathrm{mM} \mathrm{NaCl}$. (For instance: dilute $1 \mu \mathrm{L}$ of $100 \mathrm{mM}$ trans-azoTAB in $17 \mu \mathrm{L}$ of water in an Eppendorf tube, then add $1 \mu \mathrm{L}$ of $2 \mathrm{M} \mathrm{NaCl}$ followed by addition of $1 \mu \mathrm{L} 100 \mathrm{mM} d s \mathrm{DNA}$ ). Each addition should be followed by pipetting to ensure proper mixing. Upon addition of the last component $(d s \mathrm{DNA})$, the solution should become turbid, which is indicative of the formation of coacervate droplets.

2. Leave in the Eppendorf tube for ca. 5 min. This allows the droplets to grow in size by coalescence. 


\subsection{Characterization of photoswitchable coacervates under global irradiation}

Turbidity measurements and dynamic light scattering (DLS) can be used to characterize the equilibrium and kinetics behaviour of photoswitchable coacervation under global irradiation of the droplets suspension (Figure 1B). The light-modulated sequestration of fluorescently labelled solutes and dyes can be quantified by UV/vis spectrophotometry. These studies are detailed below.

\subsubsection{Equipment}

1. Eppendorf tubes, micropipettes and pipette tips

2. Low volume plastic cuvettes (minimum volume $\sim 70 \mu \mathrm{L}$ ) of $1 \mathrm{~cm}$ pathlength

3. Zetasizer Nano-ZS instrument equipped with a $633 \mathrm{~nm}$ laser (Malvern)

4. UV/vis spectrophotometer

5. UV (365 $\mathrm{nm})$ and blue $(450 \mathrm{~nm})$ LEDs

\subsubsection{Protocols}

\section{General protocol for equilibrium DLS and turbidity measurements}

The general procedure for equilibrium measurements consists in producing a suspension of trans-azoTAB/dsDNA coacervate droplets in the dark, measuring the desired property (turbidity or hydrodynamic radius), then irradiating the sample with UV (or blue) light to reach equilibrium and monitor again the desired property. These equilibrium studies are detailed below. NB: although not detailed here, turbidity and DLS equilibrium studies can also be used to screen the influence of the trans-azoTAB/dsDNA ratio and the ionic strength on the phase separation, in order to determine the optimal ratio, as well as the critical salt concentration at which coacervate disassemble.

1. Prepare a fresh trans-azoTAB/dsDNA droplets suspension $(5 \mathrm{mM}$ trans-azoTAB, $5 \mathrm{mM}$ $d s \mathrm{DNA}, 100 \mathrm{mM} \mathrm{NaCl}$, see 4.1.3.) and incubate in a low volume plastic cuvette for ca. 15 minutes.

2. Measure the average hydrodynamic radius using a Zetasizer Nano-ZS instrument equipped with a $633 \mathrm{~nm}$ laser, or measure the absorbance at $700 \mathrm{~nm}$ using a UV/vis spectrophotometer. NB: for DLS measurements, the focal point of the laser should be adjusted to be as close as possible to the inner cuvette wall to limit multiple scattering in the turbid droplet suspension, and the backscattering detector should be used; for turbidity measurements, the absorbance is measured at a wavelength at which neither trans- nor cisazoTAB absorb $(700 \mathrm{~nm})$. The absorbance of the droplets suspension should be $>1$.

3. Irradiate the suspension for ca. $15 \mathrm{~min}$ from the side of the cuvette using a UV LED operating at $365 \mathrm{~nm}$ and by positioning the sample along the emission axis at a distance of $5 \mathrm{~cm}$ from the light source. Gentle mixing by taping or pipeting should be done regularly to ensure homogeneous irradiation. The solution should become clear, indicative of the dissolution of coacervate micro-droplets.

4. Measure the average hydrodynamic radius and the absorbance. The absorbance of the clear solution should be $\sim 0$.

5. Repeat steps 3. and 4. using a blue LED operating at $450 \mathrm{~nm}$. The solution should become turbid under blue light, consistent with the reformation of coacervate microdroplets. The absorbance of the droplets suspension should be $>1$.

6. Steps 3. to 5 . can be repeated several times to demonstrate that the process is repeatable and reversible and does not show any apparent fatigue. 
Time-dependent turbidity measurements can also be performed under in situ UV or blue light irradiation to assess the kinetics of the photoswitchable phase separation behaviour.

1. Prepare a fresh trans-azoTAB/dsDNA droplets suspension (5 mM trans-azoTAB, $5 \mathrm{mM}$ dsDNA, $100 \mathrm{mM} \mathrm{NaCl}$, see 4.1.3.) and incubate in a low volume plastic cuvette for ca. 15 minutes.

2. Place the cuvette in a UV/vis spectrophotometer and start recording the evolution of the absorbance at $700 \mathrm{~nm}$ over time under magnetic stirring.

3. Irradiate the sample from the top using a UV LED (365 nm) and keep monitoring the decrease in absorbance at $700 \mathrm{~nm}$ until it reaches a plateau. NB: to ensure that the presence of the LED does not perturb the signal (e.g. if the lid of the UV/vis spectrophotometer needs to remain open when operating the LED), a blank using Milli-Q water should be first performed in the same conditions.

4. Change the irradiation wavelength to $450 \mathrm{~nm}$ (blue LED) and monitor the increase in absorbance at $700 \mathrm{~nm}$ over time until it reaches a plateau.

5. Steps 3. and 4. can be repeated at different light intensity. The characteristic time of droplet formation or dissolution under UV or blue light, respectively, is inversely proportional to the light intensity, as expected from the photo-isomerisation of azoTAB.

6. Instead of using blue light in step 4. to induce the formation of coacervate droplets, the UV-adapted sample can also be stored in the dark at a fixed temperature. Cis-trans thermal relaxation indeed occurs in the dark, leading to the spontaneous formation of coacervate droplets over time (which can be observed by recording the absorbance at $700 \mathrm{~nm}$ over time in the dark). Since thermal relaxation is enhanced at higher temperatures, the kinetics of droplets formation can be controlled by varying the temperature of the sample, providing a simple approach for the temporal programming of coacervate assembly (Figure 1A) (Martin et al., 2019b).

\section{Quantification of light-actuated capture and release of fluorescent solutes}

The light-dependent amount of fluorescent solutes or dyes sequestered in the droplets are quantified by UV/vis spectrophotometry. These studies require the use of fluorophores/dyes that have a maximum absorption wavelength higher than $550 \mathrm{~nm}$ to avoid overlapping with azoTAB absorption range.

1. Prepare $100 \mu \mathrm{L}$ of a fresh trans-azoTAB/dsDNA droplets suspension (5 $\mathrm{mM}$ transazoTAB, $5 \mathrm{mM}$ dsDNA, $100 \mathrm{mM} \mathrm{NaCl}$, see 4.1.3.) and incubate in an Eppendorf tube for ca. 10 minutes.

2. Add a small volume of a stock solution of fluorescent solute or dye to the suspension of coacervate droplets to reach a final fluorophore/dye concentration of $\sim 20 \mu \mathrm{M}$, and incubate for $\sim 5 \mathrm{~min}$. NB: the fluorophore/dye concentration should be sufficiently high to give a measurable signal by UV/vis spectrophotometry (this will depend on the molar extinction coefficient). To avoid diluting the droplets, use a relatively concentrated stock solution of solute.

3. Take a $30 \mu \mathrm{L}$ aliquot of the coacervate droplets suspension and centrifuge at $\sim 20,000 \times g$ for 10 minutes. Collect the supernatant and record the UV/vis spectrum after dilution in water to get a maximum absorption peak comprised between 0 and 1 . From that maximum absorption, the concentration of free fluorophore/dye in the supernatant can be determined. 4. Irradiate the remaining $70 \mu \mathrm{L}$ of coacervate droplets suspension with UV light, then repeat step 3. to get the concentration of free fluorophore/dye in the UV-adapted sample.

5. Irradiate the remaining $40 \mu \mathrm{L}$ of coacervate droplets suspension with blue light, then repeat step 3. to get the concentration of free fluorophore/dye in the blue-adapted sample. 6 . Kinetics studies of dye release and capture can also be performed by preparing a larger volume of coacervate droplets suspension, and taking an aliquot at different time during UV or blue light exposure to perform steps 3 . or 4 . at various times. 


\subsection{Characterization of photoswitchable coacervates under local irradiation}

Photoswitchable coacervate droplets can be observed and characterized under local irradiation by optical and confocal fluorescence microscopy. The droplets tend to adhere and spread on untreated glass coverslips, therefore a surface treatment has to be performed to avoid wetting and maintain the spherical shape of the droplets. We detail below how microscopy observations of coacervate droplets and their photoswitchable behaviour are performed.

\subsubsection{Equipment}

1. Glass slides $(\sim 76 \times 26 \times 1 \mathrm{~mm})$ and coverslips $(24 \times 24 \mathrm{~mm}, 0.13-0.17 \mathrm{~mm}$ thickness $)$

2. Coverslips staining jars (DWK Life Sciences Wheaton ${ }^{\mathrm{TM}}$ Columbia Jars).

3. UV-curing optical adhesives (NOA63 and NOA81 from Thorlabs)

4. Compressed air.

5. Inverted epifluorescence optical microscope with a $\sim 60 \times$ oil immersion objective equipped for UV and blue light excitation with a $\mathrm{Hg}$ arc lamp or LEDs (and appropriate filter cubes to select the desired wavelengths), and for monitoring red fluorescence.

6 . Confocal fluorescence microscope with a $\sim 60 \times$ oil immersion objective equipped for UV $(405 \mathrm{~nm})$ and blue light $(488 \mathrm{~nm})$ excitation, and for monitoring red fluorescence.

\subsubsection{Chemicals}

1. 3-[methoxy(polyethyleneoxy)propyl]tri-methoxysilane; 90\%, 6-9 PE units (abcr $\mathrm{GmbH}$, reference $A B 111226)$

2. Ethanol

3. Toluene

\subsubsection{Protocol}

\section{Preparation of PEGylated capillary chambers}

1. Wash coverslips with ethanol and dry using compressed air.

2. In a fume hood, place the ethanol-rinsed coverslips into a staining jar containing $5 \% \mathrm{v} / \mathrm{v}$ of 3-[methoxy(polyethyleneoxy)propyl]tri-methoxysilane diluted in toluene.

3. Incubate for $24-48 \mathrm{~h}$ at room temperature.

4. Rinse the coverslips with toluene, ethanol then water (NB: avoid touching the surface of the coverslips), and dry with compressed air.

5. To assemble the capillary chambers: first, using the UV-curing glue NOA81, glue two glass coverslips at each edge of a glass slide; then, using the UV-curing glue NOA63, glue a PEG-functionalized coverslip on top of the two other coverslips to make a capillary chamber (as shown in Figure 1C).

\section{Local photocontrol of coacervate micro-droplets}

1. Load $5 \mu \mathrm{L}$ of a droplets suspension (see 4.1.3.) into a pre-assembled capillary channel and let the coacervate droplets settle on the PEG-functionalized coverslip for $\sim 5 \mathrm{~min}$.

2. Image the droplets through the PEG-functionalized coverslip by optical microscopy using an inverted microscope and a $\sim 60 \times$ oil immersion objective. The droplets should remain spherical after settling on the coverslip. NB: Fusion and relaxation to a single droplet may be observed when two droplets come into contact, consistent with the liquid-like nature of the droplets. A movie of this process can be recorded, and the kinetics of fusion and relaxation exploited to determine the inverse capillary viscosity $(\eta / \mathrm{Y})$ of the droplets (Mitrea et al., 2018). 
3. To disassemble the droplets in situ, use the irradiation source directly installed on the microscope with appropriate filter cubes to select a wavelength range centred on $\sim 365 \mathrm{~nm}$. Simultaneous bright-field illumination (using a red filter to avoid altering the trans/cisazoTAB equilibrium composition) should be used to observe and record in real time the dissolution of droplets. Alternatively, an external light source can be used to disassemble the droplets under bright-field observation.

4. To reversibly form coacervate droplets locally, use the irradiation source directly installed on the microscope and appropriate filter cubes to select a wavelength range centred on $\sim 450 \mathrm{~nm}$. Here again, simultaneous bright-field illumination (using a red filter to avoid altering the trans/cis-azoTAB equilibrium composition) should be used to observe and record in real time the formation of droplets. Alternatively, an external light source can be used to reassemble the droplets under bright-field observation. NB: the droplets formed under blue light irradiation are smaller than the dark-adapted droplets due to the lower fraction of trans-azoTAB produced under blue light. When the blue light is turned off, coalescence between droplets can favour the formation of larger droplets that settle on the glass coverslip.

\section{Light-induced release and uptake of fluorescently labelled solutes}

Epifluorescence or confocal fluorescence microscopy can be used to assess the sequestration of fluorescent solutes and their release and capture upon light-actuated dissolution and reformation of coacervate micro-droplets. For these studies, the use of redemitting fluorophores $\left(\lambda_{\mathrm{ex}, \mathrm{em}}>550 \mathrm{~nm}\right.$ ) is highly preferred to ensure that the excitation and emission spectra of the fluorophore do not overlap with the absorption of azoTAB.

1. Prepare a fresh trans-azoTAB/dsDNA droplets suspension $(5 \mathrm{mM}$ trans-azoTAB, $5 \mathrm{mM}$ dsDNA, $100 \mathrm{mM} \mathrm{NaCl}$, see 4.1.3.) and incubate in an Eppendorf tube for ca. 10 minutes.

2. Add a small volume of a stock solution of fluorescent solute to the suspension of coacervate droplets and incubate for $\sim 5 \mathrm{~min}$ to reach a final fluorophore concentration of ca. 5. $\mu \mathrm{M}$. NB: to avoid diluting the droplets, use a relatively concentrated stock solution of solute.

3. Image the droplets by epifluorescence or confocal fluorescence microscopy using the appropriate excitation wavelength and wavelength emission range. Confocal fluorescence images can be used to determine the partition coefficient of the fluorescent solute in the coacervate phase as the ratio of the average fluorescence intensity per pixel in the coacervate droplets to the average fluorescence intensity per pixel in the background. NB: this analysis assumes that the fluorescence quantum yield of the fluorophore is the same in the coacervate phase and in the continuous aqueous phase.

4. Irradiate the sample with UV $(365 \mathrm{~nm})$ using the light source directly installed on the microscope, and acquire images. The fluorescence should become homogeneous throughout the images due to the dissolution of the coacervate droplets and associated release of the fluorescent molecules. NB: although it is not the optimal wavelength for transcis azoTAB photo-isomerisation, the $405 \mathrm{~nm}$ source can be used for UV irradiation on the confocal microscope.

5. Irradiate the sample with blue light $(450 \mathrm{~nm})$ using the light source directly installed on the microscope (and appropriate filter cubes), and acquire images. Coacervate droplets should reform, and recapture the fluorescent molecules. NB: quantification of this lightinduced release and capture process can be performed under global irradiation by UV/vis spectrophotometry (see 4.2.2.).

6. Coacervate dissolution can also be performed at the single droplet level using the FRAP module on the confocal microscope. For this purpose, load a suspension of transazoTAB/dsDNA coacervate droplets in a capillary chamber and wait for the droplets to settle on the PEGylated coverslip for $\sim 5 \mathrm{~min}$. Using the FRAP module, define a region of interest around a single droplet and irradiate with $405 \mathrm{~nm}$. The irradiation should be sufficiently long 
and intense to selectively disassemble the target droplet, but not too strong nor intense to avoid perturbing the neighbouring droplets. This approach is used to release sequestered solutes locally around the disassembled droplet (Martin et al, 2019b).

\section{Conclusions}

In this chapter, we have described a new strategy to produce photoswitchable coacervate micro-droplets capable of being reversibly dissolved with light. The droplets are formed by associative liquid-liquid phase separation between short $d s D N A$ and a cataionic azobenzene photoswitch. The latter undergoes trans-cis photoisomerisation under UV light, converting the coacervate-forming trans-azoTAB into the coacervate-inactive cis-azoTAB species. The reversible process is activated with blue light irradiation. Significantly, the dynamics of light-induced droplet dissolution and reformation correlates with the release and recapture of guest solutes. This photoswitchable coacervate platform should therefore inspire the development of new approaches for the spatiotemporal control of biomolecule compartmentalization, and open perspectives for the regulation of signalling pathways in synthetic protocells and artificial membraneless organelles.

\section{References}

Aumiller Jr, W. M., \& Keating, C. D. (2016). Phosphorylation-mediated RNA/peptide complex coacervation as a model for intracellular liquid organelles. Nature Chemistry, 8, 129-137.

Berry, J., Brangwynne, C. P., Haataja, M. Physical principles of intracellular organization via active and passive phase transitions. (2018) Reports on Progress in Physics, 81, 0406601.

Brangwynne, C. P., Tompa, P., \& Pappu, R. V. (2015). Polymer physics of intracellular phase separation. Nature Physics, 11, 899-904.

Chevallier, E., Mamane, A., Stone, H. A., Tribet, C., Lequeux, F., \& Monteux, C. (2011). Pumping-out photosurfactants from an air-water interface using light. Soft Matter, 7, 7866-7874.

Crowe, C. D., \& Keating, C. D. (2018). Liquid-liquid phase separation in artificial cells. Interface Focus, 8 , 20180032.

Deshpande, S., Brandenburg, F., Lau, A., Last, M. G. F., Spoelstra, W. K., Reese, L., et al. (2019). Spatiotemporal control of coacervate formation within liposomes. Nature Communications, 10, 1800.

Douliez, J.-P., Martin, N., Gaillard, C., Beneyton, T., Baret, J.-C. Mann, S., et al. (2017). Catanionic coacervate droplets as a surfactant-based membrane-free protocell model. Angewewandte Chemie International Edition, 56, 13689-13693.

Diguet, A., Mani, N. K., Geoffroy, M., Sollogoub, M., \& Baigl, D. (2010). Photosensitive surfactants with various hydrophobic tail lengths for the photocontrol of genomic DNA conformation with improved efficiency. Chemistry - A European Journal, 16, 11890-11896.

Estévez-Torres, A., Crozatier, C., Diguet, A., Hara, T., Saito, H., Yoshikawa, K., et al. (2009). Sequenceindependent and reversible photocontrol of transcription/expression systems using a photosensitive nucleic acid binder. Proceedings of the National Academy of Sciences USA, 106, 12219-12223.

Hayashita, T., Kurosawa, T., Miyata, T., Tanaka, K., \& Igawa, M. (1994). Colloid and Polymer Science, 272, 1611-1619.

Hyman, A. A., Weber, C. A., \& Jülicher, F. (2014). Liquid-liquid phase separation in biology. Annual Review of Cell Developmental Biology, 30, 39-58.

Hondele, M., Sachdev, R., Heinrich, S., Wang, J., Vallotton, P., Fontoura, B. M. A., \& Veis, K. (2019). DEADbox ATPases are global regulators of phase-separated organelles. Nature, 573, 144-148.

Le Ny, A.-L. M., \& Lee, C. T. (2006). Photoreversible DNA condensation using light-responsive surfactants. Journal of the Americal Chemical Society, 128, 6400-6408.

Love, C., Steinkühler, J., Gonzales, D. T., Yandrapalli, N., Robinson, T., Dimova, R., et al. (2020). Angewandte Chemie International Edition, 59, 5950-5957.

Martin, N., Li, M., \& Mann, S. (2016). Selective uptake and refolding of globular proteins in coacervate microdroplets. Langmuir, 32, 5881-5889.

Martin, N., Sharma, K. P., Harniman, R. L., Richardson, R. M., Hutchings, R. J., Alibhai, D., et al. (2017). Lightinduced dynamic shaping and self-division of multipodal polyelectrolyte-surfactant microarchitectures via azobenzene photomechanics. Scientific Reports, 7, 41327.

Martin, N., Douliez, J.-P., Qiao, Y., Booth, R., Li, M. \& Mann, S. (2018). Antagonistic chemical coupling in selfreconfigurable host-guest protocells. Nature Communications, 9, 3652. 
Martin, N. (2019a). Dynamic synthetic cells based on liquid-liquid phase separation. ChemBioChem, 20, 25532568.

Martin, N., Tian, L., Spencer, D., Coutable-Pennarun, A., Anderson, J. L. R., \& Mann, S. (2019b). Photoswitchable phase separation and oligonucleotide trafficking in DNA coacervate microdroplets. Angewandte Chemie International Edition, 58, 14594-14598.

Mitrea, D. M., Chandra, B., Ferrolino, M. C., Gibbs, E. B., Tolbert, M., White, M. R., et al. (2018). Methods for physical characterization of phase separated bodies and membraneless organelles, Journal of Molecular Biology, 430, 4773-4805.

Nakashima, K. K., Baaji, J. F., \& Spruijt, E. (2018). Reversible generation of coacervate droplets in an enzymatic network. Soft Matter, 14, 361-367.

Nakashima, K. K., Vibhute, M. A., \& Spruijt, E. (2019). Biomolecular chemistry in liquid phase separated compartments. Frontiers in Molecular Biosciences, 6, 21.

Nott, T. J., Petsalaki, E., Faber, P., Jervis, D., Fussner, E., Plochowietz, A., et al. (2015). Phase transition of a disordered nuage protein generates environmentally responsive membraneless organelles. Molecular Cell, 57, 936-947.

Perry, S. L., Li, Y., Priftis, D., Leon, L., \& Tirrell, M. (2014). The effect of salt on the complex coacervation of vinyl polyelectrolytes. Polymers, 6, 1756-1772.

Perry, S. L. (2019). Phase separation: bridging polymer physics and biology. Current Opinion in Colloid \& Interface Science, 39, 86-97.

Rai, A. K., Chen, J., Selbach, M., \& Pelkmans, L. (2018); Kinase-controlled phase transition of membraneless organelles in mitosis. Nature, 559, $211-216$

Shin, Y., Brangwynne, C. P. (2017). Liquid phase condensation in cell physiology and disease. Science. 357, eaaf4382.

Shin, Y., Berry, J., Pannucci, N., Haataja, M. P., Toettcher, J. E., Brangwynne, C. P. (2017). Spatiotemporal control of intracellular phase transitions using light-activated optodroplets. Cell, 168, 159-171.

Sollogoub, M., Guieu, S., Geoffroy, M., Yamada, A., Estévez-Torres, A., Yoshikawa, K., et al. (2008). Photocontrol of single-chain DNA conformation in cell-mimicking microcompartments, ChemBioChem, 9, 1201-1206.

Wheeler, R. J., \& Hyman, A. A. (2018). Controlling compartmentalization by non-membrane-bound organelles. Philosophical Transactions of the Royal Society B, 373, 20170193.

\section{Acknowledgements}

We acknowledge funding from IdEx Bordeaux (ANR-10-IDEX-03-02), an Investissement d'Avenir program of the French government, managed by the Agence Nationale de la Recherche. 